\title{
EXPERIMENTOS EM PARCELAS SUBDIVIDIDAS COM TRATAMENTOS PRIMÁRIOS EM BLOCOS INCOMPLETOS PARCIALMENTE BALANCEADOS I: UMA SOLUÇÃO DAS EQUAÇÕES NORMAISı
}

\author{
A.R. DE MORAIS ; M.C. STOLF NOGUEIRA ${ }^{3}$ \\ 2 Depto. de Ciências Exatas - UFLA, C.P. 37, CEP: 37200-000 - Lavras, MG; \\ ${ }^{3}$ Depto. de Matemática e Estatistica - ESALQUSP C.P. 9, CEP: 13419-900 - Piracicaba, SP
}

RESUMO: O objetivo deste trabalho fol realizar um estudo para obtençāo de uma solução adequada das equaçóes normais de experimentos em parcelas subdivididas com tratamentos primários dispostos segundo uma estrutura de blocos incompletos parcialmente balanceados.

Descritores: delineamento experimental, equaçōes normais, blocos incompletos, parcelas subdivididas.

\section{SPLIT-PLOT EXPERIMENTS WITH MAIN TREATMENTS IN PARTIALLY BALANCED INCOMPLETE BLOCKS I: A SOLUTION OF THE NORMAL EQUATIONS}

ABSTRACT: The objective of this study was to develop a suitable methodology for the solution of normal equations in split-plot experiments involving two-way treatment structures when the design for the whole plot experimental units is a partially balanced incomplete block design.

Key Words: Experimental design, normal equations, incomplete blocks, split- plot.

\section{INTRODUÇĀO}

Um dos problemas fundamentais no planejamento de experimentos, está na escolha correta do tipo de delineamento que melhor se adapte às condições experimentais específicas do problema em estudo. $\mathrm{Na}$ experimentação, quando se têm dois ou mais fatores para serem estudados simultaneamente, uma das opçð̃es é a utilização do experimento em parcelas subdivididas.

$O$ experimento em parcelas subdivididas, segundo MILLIKEN \& JOHNSON (1984), envolve uma estrutura de tratamentos com dois ou mais fatores e uma estrutura de delineamento em blocos incompletos, apresentando, pelo menos, dois tamanhos diferentes de unidades experimentais: as unidades experimentais grandes, chamadas de parcelas primárias ou parcelas $e$ as unidades menores, chamadas de subparcelas. Nas parcelas são casualizados os tratamentos primários e nas subparcelas os tratamentos secundários.

Esse tipo de experimento largamente utilizado nas pesquisas agrícolas, industrial e biológica, é útil em situações, tais como: a) quando os níveis de um ou mais fatores exigem grandes quantidades do material experimental (por exemplo, métodos de preparo do solo); b) quando informações prévias asseguram que as diferenças entre os niveis de um dos fatores são maiores do que às do outro fator, $c$ ) quando se deseja maior precisão para comparações entre níveis de um dos fatores; d) quando existe um fator de maior importância e outro de importância secundária, sendo que este é incluído para aumentar a extensão dos resultados e e) nas situações práticas onde é dificil a instalação do experimento no esquema fatorial. Uma farta bibliografia sobre o assunto, sua aplicação e a metodologia de análise está ressaltada em diversos livros textos, tais como, LEONARD

\footnotetext{
${ }^{1}$ Parte da tese apresentada a Escola Superior de Agricultura "Luiz de Queiroz" pelo primeiro autor.
} 
\& CLARK (1939), KEMPTHORNE (1952), COCHRAN \& COX (1957), STEEL \& TORRIE (1960), HICKS (1973) e PIMENTEL GOMES (1985), dentre outros.

$O$ caso de experimentos em parcelas subdivididas quando os tratamentos primários estão dispostos na estrutura de delineamento em blocos incompletos balanceados (BIB) foi abordado por IEMMA (1981), que ressaltou existir uma lacuna quanto a esses experimentos em blocos incompletos. Entretanto, existem situaçðes em que o delineamento em BIB, nem sempre é viável, pois pode exigir um número elevado de repetiçzes, tornando-se praticamente impossivel sua utilização. Segundo BOSE \& NAIR (1939), um delineamento em blocos incompletos é parcialmente balanceado se: $v$ tratamentos são arranjados em b blocos de $k$ unidades com diferentes tratamentos; cada tratamento ocorre em $r$ blocos: tomando-se um tratamento qualquer os demais podem ser divididos em $m$ grupos de tamanhos $n_{1}, \ldots, n_{m}$ de tal forma que os tratamentos do iésimo grupo ocorram juntos com o dado tratamento em $\lambda_{1}$ blocos; se o tratamento $A$ é i-ésimo associado de $B$, então o tratamento $B$ é i-ésimo associado de A. Se A e B são i-ésimos associados, então o número de tratamentos que são comuns aos j-ésimos associados de A e aos k-ésimos associados de B é p $p_{j k}^{i}$ e eles são independentes do par de tratamentos considerado.

O presente trabalho tem como objetivo apresentar um estudo para experimentos em parcelas subdivididas quando os tratamentos primários estão dispostos segundo uma estrutura de delineamento em blocos incompletos parcialmente balanceados, no que se refere a obtenção de soluções para os efeitos de tratamentos.

\section{METODOLOGIA}

Para o desenvolvimento da metodologia, adotou-se o seguinte modelo linear:

$$
y_{1 j !}=\mu+b_{j}+t_{1}+t_{1}^{*}+\delta_{1}+\varepsilon_{1,1}
$$

onde com: $i=1,2, \ldots, v$ tratamentos primários; $j=$ $1,2, \ldots$ b blocos; $1=1,2, \ldots$, u tratamentos secundários; $y_{\text {IJ }}$ é o valor observado na subparcela correspon- dente ao l-ésimo tratamento secundário, dentro do iésimo tratamento primário, no j-ésimo bloco; u é uma constante que representa a média geral; $b_{j}$ é o efeito do j-ésimo bloco; t ć o efeito do i-ésimo tratamento primário; $t *$ á o efeito do l-ésimo tratamento secundário; $\delta_{\mathrm{il}}$ c o efeito de interação entre o i-ésimo tratamento primário e o l-ésimo tratamento secundário; $\varepsilon_{\mathrm{jil}}$ é o erro aleatório atribuido a observação $y_{1 \mathrm{jl}}$, considerado como o componente do resíduo.

$\mathrm{Na}$ forma matricial, o modelo linear é dado por:

$$
y=x \theta+\varepsilon
$$

onde: y é um vetor de realizaçóes de variáveis aleatórias, com dimensø̃es (vru) $x$ (1); X é uma matriz dos coeficientes dos parâmetros do modelo (matriz do delineamento), com dimensões (vru) $x$ $(1+v+b+u+v u) ; \theta$ é um vetor de parâmetros desconhecidos do modelo, com dimensões $(1+v+b+u+v u)$ $\mathrm{x}(1) ; \varepsilon$ ć um vetor de variáveis aleatórias não observáveis, com dimensões (vru) x (1), as quais são assumidas serem independentes e normalmente distribuidas com $\varepsilon \cap \mathrm{N}(\varnothing, \Sigma)$, cuja estrutura de erros é dada por:

$\operatorname{Cov}\left(y_{i l} ; y_{i j^{\prime \prime}}\right)= \begin{cases}\sigma_{\mathrm{a}}^{2}+\sigma_{b}^{2} & \text { se } i=i^{\prime} ; j=j^{\prime} \text { el }=l^{\prime} \\ \sigma_{\mathrm{a}}^{2} & \text { se } \mathrm{i}=\mathrm{i}^{\prime} ; \mathrm{j}=\mathrm{j}^{\prime} \text { el } \neq \mathrm{l}^{\prime} \\ 0 & \text { em caso contrário }\end{cases}$

Conforme PIMENTEL GOMES (1968) e IEMMA (1981), efetuou-se a partição da matriz X, da seguinte maneira:

$$
X=\left[X_{1}: X_{2}: X_{3}: X_{4}: X_{5}\right]
$$

onde: $X_{1}$ é o vetor dos coeficientes associados à constante $\mu$ de dimensões (vru) x (1); $X_{2}$ é a matriz dos coeficientes associados aos blocos, de dimensбes ( $v r u) \times(b) ; X_{3}$ e a matriz dos coeficientes associados aos tratamentos primários, de dimensões (vru) $x(v) ; X_{4}$ é a matriz dos coeficientes associados aos tratamentos secundários, de dimensões 
(vru) $\mathrm{x}(\mathrm{u}) ; \mathrm{X}_{5}$ é a matriz dos coeficientes associados às interaçð̃es $\delta_{\mathfrak{v}}$, de dimensðes (vru) $x$ (vu).

A partição do vetor dos parâmetros $\theta$ correspondente à partição da matriz $\mathrm{X}$ é dada por:

onde,

$$
\theta^{\varsigma}=\left[\mu: \beta^{\varsigma}: \tau^{\varsigma}: \tau^{*}: \delta^{\varsigma}\right]
$$

$\beta=\left[\begin{array}{l}b_{1} \\ b_{2} \\ \ldots \\ b_{b}\end{array}\right]_{1} ; \tau=\left[\begin{array}{c}t_{1}^{\prime} \\ t_{2} \\ \ldots \\ t_{v}\end{array}\right]_{1} ; \tau^{*}=\left[\begin{array}{c}t_{1}^{*} \\ t_{2}^{*} \\ \ldots \\ t_{u}^{*}\end{array}\right]_{1} \delta=\left[\begin{array}{l}\delta_{11} \\ \delta_{12} \\ \ldots \\ \delta v u\end{array}\right]_{1}$

\section{Sistema de equações normais}

Aplicando o método de mínimos quadrados, determinou-se o sistema de equaçðes normais (SEN): $X^{\prime} X \theta=X^{\prime} y, o$ qual resultou em

$$
\begin{aligned}
& {\left[\begin{array}{lllll}
X_{1}^{\prime} X_{1} & X_{1}^{\prime} X_{2} & X_{1}^{\prime} X_{3} & X_{1}^{\prime} X_{4} & X_{1}^{\prime} X_{5} \\
X_{2} X_{1} & X_{2}^{\prime} X_{2} & X_{2}^{\prime} X_{3} & X_{2}^{\prime} X_{4} & X_{2}^{\prime} X_{5} \\
X_{3}^{\prime} X_{1} & X_{3}^{\prime} X_{2} & X_{3}^{\prime} X_{3} & X_{3}^{\prime} X_{4} & X_{3}^{\prime} X_{5} \\
X_{4} X_{1} & X_{4}^{\prime} X_{2} & X_{4} X_{3} & X_{4}^{\prime} X_{4} & X_{4} X_{5} \\
X_{5}^{\prime} X_{1} & X_{5}^{\prime} X_{2} & X_{5}^{\prime} X_{3} & X_{5}^{\prime} X_{4} & X_{5}^{\prime} X_{5}
\end{array}\right] \cdot} \\
& {\left[\begin{array}{l}
\mu \\
\beta \\
\tau \\
\tau \\
\delta
\end{array}\right]=\left[\begin{array}{l}
X_{1}^{\prime} y \\
X_{2}^{\prime} y \\
X_{3}^{\prime} y \\
X_{4}^{\prime} y \\
X_{5}^{\prime} y
\end{array}\right]}
\end{aligned}
$$

onde as submatrizes são designadas por: $X^{\prime}, X_{1}$ é uma submatriz, de dimensøes (1) x (1), constituida por $b k u=v r u=n$ que é o número total de unidades experimentais ou de subparcelas; $X^{\prime}{ }_{1} X_{2}$ é uma submatriz, de dimensões (1) $x$ (b), representada por $k u, E_{b}$, correspondente ao vetor associado ao número de subparcelas em cada bloco, onde $\mathrm{E}$ é um vetor de uns; $X_{1}{ }_{1} X_{3}$ é uma submatriz, de dimensð̃es (1) $X$ (v), representada por $r{ }_{1} E_{v}$, correspondente ao vetor associado ao número de repetiçóes dos tratamentos primários, onde $\mathrm{E}$ é um vetor de uns; $\mathrm{X}_{1}{ }_{1} \mathrm{X}_{4}$ é uma submatriz, de dimensø̃es (1) $\times(u)$, representada por $\mathrm{vr}_{1} \mathrm{E}_{u}$, correspondente ao vetor associado ao número de repetições dos tratamentos secundários, onde $E$ é um vetor de uns; $X^{\prime}{ }_{1} X_{5}$ é uma submatriz de dimensões (1) $x(v u)$, representada por $r_{1} E_{v u}$ correspondente ao vetor associado ao número de repetiçós das interações, onde $\mathrm{E}$ é um vetor de uns; $\mathrm{X}_{2}$ $\mathrm{X}_{2}$ é uma submatriz diagonal $\{\mathrm{ku}, \mathrm{ku}, \ldots, \mathrm{ku}\}$, de dimensões (b) $x$ (b), representada por $K$, correspondente a matriz do número de subparcelas em cada bloco; $\mathrm{X}_{2} \mathrm{X}_{4}$ é uma submatriz, de dimensões (b) $\mathrm{X}$ (u), representada por $A=k_{b} E_{u}$, correspondente $a$ matriz do número de tratamentos primários por bloco, onde $\mathrm{E}$ é uma submatriz de uns; $\mathrm{X}_{2}{ }_{2} \mathrm{X}_{\mathrm{s}}$ é uma submatriz, de dimensões (b) $x(\mathrm{vu})$, representada por $\mathrm{V}$, correspondente a matriz de incidência da interação $\delta_{\mathfrak{j}}$ no bloco j, constituida por $v_{1, j}$, onde:

$V_{j, i l}=\left\{\begin{array}{l}1, \text { se a interação } \delta_{1 l} \text { ocorre no bloco } \mathrm{j} ; \\ 0, \text { caso contrário; }\end{array}\right.$

$\mathrm{X}_{3}{ }_{3} \mathrm{X}_{2}$ é uma submatriz, de dimensões (v) $\mathrm{x}$ (b), correspondente a matriz de incidência do i-ésimo tratamento primário no j-ésimo bloco, denominada $\mathrm{N}$, cujos elementos $n_{j j}$ que a compõem, são:

$N_{i j}=\left\{\begin{array}{l}\mathrm{u}, \text { se o tratamento principal i ocorre np bloco j } \\ 0, \text { caso contrário; }\end{array}\right.$

$\mathrm{X}_{3}{ }_{3} \mathrm{X}_{3}$ é uma submatriz diagonal $\{\mathrm{ru}, \mathrm{ru}, \ldots, \mathrm{ru}\}$, de dimensões (v) $x$ (v), representada por $R$, correspondente ao número de repetiçóes de cada tratamento primário; $X_{3}{ }_{3} X_{4}$ é uma submatriz, de dimensóes (v) $X$ (u), correspondente ao número de repetições de cada par $\delta_{i 1}$ da interação representada por $P=r_{v} E_{u}$, onde $E$ é uma submatriz de uns, $X_{3}{ }^{\prime} X_{5}$ é uma submatriz, de dimens $\tilde{e}$ es ( $v) \times(v u)$, representada por $S$, correspondente a matriz de incidência dos tratamentos primários nos, pares $\delta_{\mathrm{il}}$ onde: 


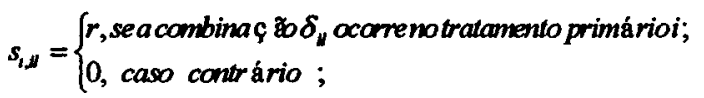

$\mathrm{X}_{4}{ }^{\prime} \mathrm{X}_{4}$ é uma submatriz diagonal $\{\mathrm{vr}, \mathrm{vr}, \ldots \mathrm{vr}\}$, de dimensôes $(u) \times(u)$, correspondente ao número de repetiçðes dos tratamentos secundários, representada por $U ; X_{4}$ 'X, é uma submatriz, de dimensóes (u) $x(v u)$, representada por $H=\left[r I_{(u)}: r I_{(u)}: \ldots: r I_{(u)}\right.$ ] e correspondente a matriz de incidência dos tratamentos secundários nos pares $\left(\mathfrak{t t}^{\prime}\right)_{1}$ onde I é uma matriz identidade de dimensões (u) $x(u) ; X_{s} X_{s}$ é uma submatriz diagonal $\{r, r, \ldots, r\}$ de dimensões (vu) $x$ (vu), associada ao número de repetiçóes dos pares $\delta_{11}$ da interação, representada por $L ; X_{1}^{\prime} y$ é um vetor referente ao total geral observado, de dimensóes (1) x (1), representado por $G ; X_{2}^{\prime} y$ é um vetor com os totais de blocos, de dimensões (b) $x$ (1), representado por $B$ sendo $B^{\prime}=\left[B_{1}, B_{2}, \ldots, B_{b}\right]$; $X_{3}{ }_{3} y$ é um vetor com os totais de tratamentos primários, de dimensóes $(v) \times(1)$, representado por $T$, sendo $T^{\prime}=\left[T_{1}, T_{2}, \ldots, T_{v}\right] ; X_{4}^{\prime} y$ é um vetor com os totais de tratamentos secundários, de dimensóes $(u)$ $x(1)$, representado por $T^{*}$, sendo $T^{*}=\left[T^{*}, T^{*}\right.$, $\left.\ldots, \mathrm{T}^{*}{ }_{\mathrm{u}}\right] ; \mathrm{X}_{5}{ }_{5} \mathrm{y}$ é um vetor com os totais de interação, de dimensões (vu) $x(1)$, representado por $D$, sendo $\mathrm{D}=\left[\mathrm{D}_{11}, \ldots, \mathrm{D}_{1 \mathrm{u}}, \ldots, \mathrm{D}_{\mathrm{v} 1}, \ldots, \mathrm{D}_{\mathrm{vu}}\right]$.

Desse modo, as partições efetuadas em (a.5), estão escritas na seguinte forma:

$\left[\begin{array}{ccccc}n & k u_{1} E_{b} & r u_{1} E_{v} & v_{1} E_{u} & r_{1} E_{v u} \\ k u_{b} E_{1} & K & N^{\prime} & A & V \\ r u_{v} E_{1^{\prime}}^{\prime}: & N & R & P & S \\ v r_{u} E_{1} & A^{\prime} & P^{\prime} & U & H \\ r_{v u} E_{1} & V^{\prime} & S^{\prime} & H^{\prime} & L\end{array}\right]$

$$
\left[\begin{array}{l}
\mu \\
\beta \\
\tau \\
\tau^{*} \\
\delta
\end{array}\right]=\left[\begin{array}{c}
\mathrm{G} \\
\mathrm{B} \\
\mathrm{T} \\
\mathrm{T}^{*} \\
\mathrm{D}
\end{array}\right]
$$

\section{Solução do sistema de equaçōes normais}

$\mathrm{O}$ número de equações do sistema de equaçôes normais em (a.6) é $1+b+v+u+v u$; mas, verifica-se que a soma das equaçóes $\beta$, a soma das equações $\tau$ e a soma das equações $\tau^{*}$ são idênticas à equação de $\mu$, implicando em três relações linearmente dependentes entre as linhas de $X$ 'X. Nas equações $\delta$, as somas em relação a $l$, são iguais às equações $\tau$ para $\mathrm{i}=1, \ldots, v$ representando outras $\mathrm{v}$ relações lineares entre as linhas de $\mathrm{X}$ 'X. Do mesmo modo, as somas em relação a $i$, são iguais às equações $\tau^{*}$ para $1=1, \ldots, u$; entretanto, dessas $u$ relações lineares representadas agora, somente $\mathrm{u}-1$ delas são linearmente independentes daquelas já descritas. Portanto, o número de relações lineares dependentes é $3+v+u-1=v+u+2$.

Assim, o posto de $X^{\prime} X$ é $r[X]=v u+b-1 \leq$ $\min \{n, p\}$, sendo $p$ o número de parâmetros, então as equaçóes normais de (a.5) não tem solução única. Pois, $X^{\prime} X$ é singular, e portanto não existe (X'X) e o SEN é indeterminado, mas é sempre consistente (IEMMA, 1988).

Uma solução de mínimos quadrados é $\theta^{0}=$ $\left(X^{\prime} X\right)^{0} X^{\prime} y$, para qualquer inversa generalizada de $X ' X$. Uma altemativa é a estratégia de "completar"o posto da matriz $X$. Isto tem sido feito de vários modos; dentre êles, cita-se a restrição nas soluçōes. A restrição tem como objetivo completar o posto da matriz $X ' X$, tomando-a invertível, e assim determinando um $\theta$ único.

Então, completando-se o posto da matriz $X$, com $p-r[x]=v+u+2$ linhas linearmente independentes das linhas da matriz $X$, que constituem um conjunto de funções paramétricas conjuntamente não estimáveis e independentes entre si.

Um conjunto de restrições paramétricas, pode ser formado pelas seguintes funções lineares nåo estimáveis:

$$
\begin{aligned}
& \sum_{j=1}^{b} b_{j}=\sum_{i=1}^{v} t_{1}=\sum_{l=1}^{u} t_{1}^{*}=\sum_{i=1}^{v}\left(\delta_{11}\right)= \\
& =\sum_{l=1}^{u}\left(\delta_{l l}\right)=0
\end{aligned}
$$


Ressalta-se que, se outro conjunto de restrições é adotado, obtém-se outra solução, mas $X \theta^{0}$ é invariante para qualquer $\theta^{\circ}$ solução de (a.6), segundo IEMMA (1988).

\section{PBIB com $m$ classes de associados}

De acordo com as restrições impostas em (a.7) e face às estruturas das matrizes envolvidas, $o$ SEN de (a.6) resultou em:

$$
\begin{array}{ll}
\mathrm{n} \hat{\mu} & = \\
\mathrm{ku}_{\mathrm{b}} \mathrm{E}_{1} \hat{\mu}+\mathrm{K} \hat{\beta}+\mathrm{N} \hat{\tau} & =\mathrm{B} \\
\mathrm{u}_{\mathrm{v}} \mathrm{E}_{1} \hat{\mu}+\mathrm{N} \hat{\beta}+\mathrm{R} \hat{\tau} & =T \\
\mathrm{v}_{\mathrm{v}} \mathrm{E}_{1} \hat{\mathrm{u}}+\mathrm{U} \hat{\tau} & T \\
\mathrm{r}_{\mathrm{v}} \mathrm{E}_{1} \hat{\mu}+\mathrm{V} \hat{\beta}+S \hat{\tau}+H \hat{\tau}^{*}+L \hat{\delta} & =\mathrm{D}
\end{array}
$$

onde (a.9) e (a.10) portaram-se de modo análogo ao SEN para experimentos em blocos incompletos. De (a.8) resultou $\hat{\mu}=G / n$.

\section{Estimaçāo dos efeitos de tratamentos principais}

Da teoria geral de blocos incompletos, discutida por PIMENTEL GOMES (1968), JOHN (1980), dentre outros, pode-se escrever o sistema de equações normais reduzido (SENR) ou sistema de equações normais para efeitos ajustados de tratamentos primários, a partir de (a.9) e (a.10), na forma: $\mathrm{C} \tau=\mathrm{Q}$, onde $\mathrm{C}=\mathrm{R}-\mathrm{NK}^{-1} \mathrm{~N}^{\prime}$ é uma matriz de dimensões (v) $x(v)$, com característica $v-1$, e com os seguintes elementos:

$$
c_{n^{\prime}}=\left\{\begin{array}{ll}
{[\mathrm{ru}(\mathrm{k}-1)] / \mathrm{k},} & \text { sei }=\mathrm{i}^{\prime} \\
-1 z_{n^{\prime}} / \mathrm{k}, & \text { sei } \neq \mathrm{i}^{\prime}
\end{array} \quad \mathrm{i}, \mathrm{i}^{\prime}=1,2, \ldots, \mathrm{v}\right.
$$

sendo $\lambda_{11}$ o número de vezes que os tratamentos primários i e i' ocorrem juntos no mesmo bloco; $\mathrm{Q}=\mathrm{T} \cdot \mathrm{NK}^{-\mathrm{I}} \mathrm{B}$ é o vetor de totais ajustados de tratamentos primários, e é composto pelos seguintes elementos: $Q_{1}=T_{1}-A_{1} / k$, sendo $A_{1}$ a soma dos totais dos blocos que contêm o tratamento primário $\mathrm{i}$.

Dado que a matriz $C$ é singular, o SENR possui infinitas soluções. Uma solução de mínimos quadrados, pode ser obtida com a utilização de inversas generalizadas (RAO, 1973; JOHN, 1980; IEMMA, 1988), ou introduzindo-se restrições (PIMENTEL GOMES, 1968; IEMMA, 1981; OLIVEIRA, 1985; DAS \& GIRI, 1986).

De acordo com o SENR, $C \tau=Q$, e conforme a caracterização da matriz C, dada em (a.13), para um dado tratamento primário $\mathrm{s}(\mathrm{i}=1, \ldots, \mathrm{s}, \ldots$, v) tem-se a seguinte equação normal reduzida para $\tau_{s}$ :

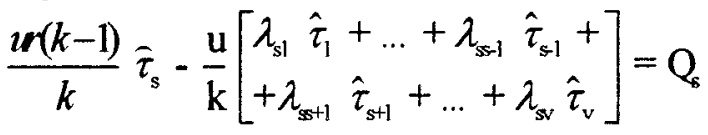
sendo $s=1,2, \ldots, v$ tratamentos primários; que resulta em:

$\mathrm{ur}(\mathrm{k}-1) \hat{\tau}_{\mathrm{s}}-\mathrm{u} \sum_{i=1}^{m} \lambda_{\mathrm{i}} \mathrm{S}_{1}\left(\hat{\tau}_{\mathrm{s}}\right)=\mathrm{KQ} \mathrm{s}$

onde $S_{1}\left(\hat{\tau}_{s}\right)$ corresponde a soma dos efeitos dos tratamentos primários que são os i-ésimos associados do tratamento s, e $\lambda_{1}$ é um dos parâmetros do primeiro tipo, definido por BOSE \& NAIR (1939), com $\mathrm{i}=1,2, \ldots, \mathrm{m}$ associados.

Verifica-se que em (a.14), o efeito do tratamento primário $\tau_{s}$ na forma como é colocado, está posto em função de seus associados, que também não são conhecidos. Mas, segundo CHAKRABARTI (1962) e MORAIS (1992), sabe-se que

$$
\operatorname{ur}(k-1) \hat{\tau}_{s}=K Q_{s}+u \sum_{j=1}^{m} S_{j}\left(Q_{s}\right) \sum_{i=1}^{m} \lambda_{1} f_{1 j}
$$


onde $S_{j}\left(Q_{g}\right)$ é a soma dos Q's dos j-ésimos associados do s-ésimo tratamento primário; e $f_{y}$ são constantes que satisfazem a:

$$
\begin{aligned}
& S_{1}\left(\hat{\tau}_{\mathrm{s}}\right)=\mathrm{f}_{\mathrm{i} 1} \mathrm{~S}_{1}\left(Q_{s}\right)+\mathrm{f}_{\mathrm{i} 2} S_{2}\left(Q_{s}\right)+\ldots \\
& +\ldots+\mathrm{f}_{\mathrm{mm}} S_{m}\left(\mathrm{Q}_{\mathrm{s}}\right) .
\end{aligned}
$$

Assim, de (a.15) uma solução para $\tau_{s}$ é:

$\hat{\tau}_{s}=\frac{1}{r(k-1)}\left[\frac{k}{u} Q_{s}+\sum_{j=1}^{m} s_{j}\left(Q_{s}\right) \sum_{i=1}^{m} \lambda_{1} f_{i j}\right]$

que é a expressão que permite obter os efeitos ajustados de tratamentos primários para um $\mathrm{PBIB}(\mathrm{m})$.

Pode-se fazer, a partir de (a.16), uma generalização para a forma matricial $\hat{\tau}=M^{-1} Q$, sendo os elementos da matriz $M^{-1}=\left\{m_{i 1},\right\}$ iguais a:

$$
m_{1^{\prime}}= \begin{cases}k /[u r(k-1)] & , \text { se } i=i^{\prime} \\ \sum_{1=1}^{m} \lambda_{1} f_{i^{\prime}} /[r(k-1)], & \text { se } i \neq i^{\prime}\end{cases}
$$

\section{Estimaçăo dos efeitos de tratamentos secundarios}

Reportando-se ao sistema de equações normais, e resolvendo-se (a.12), tem-se que as soluçбes de mínimos quadrados para os efeitos dos tratamentos secundários são determinados a partir de:

$$
\hat{\tau}^{*}=U^{-1} T^{*}-{ }_{u} E_{1} \hat{\mu}
$$

Portanto,

$$
\vec{\tau}_{1}^{*}=\frac{1}{\mathrm{vr}} \mathrm{T}_{1}^{*}-\hat{\mu}
$$

que é uma solução semelhante àquelas obtidas para tratamentos secundários nos experimentos instalados no delineamento em blocos casualizados com parcelas subdivididas, e idêntica àquela encontrada pro IEMMA (1981), para os experimentos em BIB.

\section{Estimação dos efeitos de interação}

Para a determinação das soluções dos efeitos estimados das interaçð̃es ou pares de $\delta_{11}$ substituem-se em (a.12) as experessões de
$\beta=K^{-1} B-K^{-1} N^{\prime} \hat{t}-{ }_{b} E_{1} \hat{u}$ obtida de (a.9), de $\hat{\tau}^{*}$ encontrada em (a.18), e $\hat{\tau}=R^{-1} T-R^{-1} N \beta-{ }_{v} E_{1} \hat{u}$ originada de (a.10).

Fazendo as substituiçðes, tem-se que:

$$
\begin{aligned}
& r_{w} E_{1} \hat{\mu}+\mathrm{S}^{\prime}\left(\mathrm{R}^{-1} \mathrm{~T} \cdot \mathrm{R}^{-1} \hat{N \beta}-{ }_{v} \mathrm{E}_{1} \hat{\mathrm{u}}\right)+\mathrm{V}\left(\mathrm{K}^{-1} B-\right. \\
& \left.-K^{-1} N^{\top} \hat{\tau}-{ }_{b} E_{1} \hat{\mu}\right)+\mathrm{H}\left(\mathrm{U}^{-1} T^{*}-{ }_{u} E_{1} \hat{\mu}\right)+L \hat{\delta}=D
\end{aligned}
$$

Assim, uma solução para o efeito estimado de um elemento qualquer da interação, é dada por:

$$
\widehat{\delta}_{11}=\bar{y}_{11}-\bar{y}_{1}-\bar{y}_{1}+\bar{y}
$$

que é um resultado idêntico aqueles obtidos em ensaios com parcelas subdivididas, envolvendo delineamentos em blocos casualizados. Assim, verificase que os efeitos de interação não são ajustados para blocos.

\section{PBIB com duas classes de associados}

Os delineamentos em bloco incompletos parcialmente balanceados com duas classes de associados, ou simplesmente PBIB(2), são os delineamentos de particular importância, pelo fato de serem os mais utilizados na prática, por isso merecem uma maior atenção.

Para $m=2$, de (a.15) tem-se que:

$$
\boldsymbol{m}(k-1) \hat{\tau}_{\mathrm{s}}=\left[\begin{array}{l}
\mathrm{kQ}+\mathrm{u}\left(\lambda_{1} f_{11}+\lambda_{2} \mathrm{f}_{21}\right) \mathrm{S}_{1}\left(Q_{s}\right)+ \\
+\mathrm{u}\left(\lambda_{1} \mathrm{f}_{12}+\lambda_{2} \mathrm{f}_{22}\right) \mathrm{S}_{2}\left(\mathrm{Q}_{\mathrm{s}}\right)
\end{array}\right]
$$

Assim, os elementos $f_{\mathrm{j}}(i, j=1,2)$, obtidos a partir da matriz $A$, são:

$$
\begin{gathered}
A=\left[\begin{array}{ll}
a_{11} & a_{12} \\
a_{21} & a_{22}
\end{array}\right] \Rightarrow A^{-1}=\left[\begin{array}{ll}
f_{11} & f_{12} \\
f_{21} & f_{22}
\end{array}\right]=\frac{1}{\Delta}\left[\begin{array}{cc}
a_{22} & -a_{12} \\
-a_{21} & a_{11}
\end{array}\right] \\
, \Delta=a_{11} a_{22}-a_{12} a_{21}
\end{gathered}
$$
onde:

$$
\begin{aligned}
& \text { (i) } a_{11}=\frac{u}{k}\left[r(k-1)+\left(\lambda_{1}-\lambda_{2}\right) p_{12}^{1}+\lambda_{1}\right] \\
& \text { (ii) } a_{12}=\frac{u}{k}\left(\lambda_{1}-\lambda_{2}\right) p_{12}^{2} \\
& \text { (iii) } a_{21}=\frac{u}{k}\left[\left(\lambda_{2}-\lambda_{1}\right) p_{21}^{1}\right]
\end{aligned}
$$


(iv) $a_{22}=\frac{u}{k}\left[r(k-1)+\left(\lambda_{2}-\lambda_{1}\right) p_{21}^{2}+\lambda_{2}\right]$

Substituindo tem-se que:

$\operatorname{co}(k-1) \hat{\tau}_{\mathrm{s}}=\mathrm{KQ}_{\mathrm{s}}+u\left(\lambda_{1} \mathrm{a}_{2} / \Delta-\lambda_{2} \mathrm{a}_{21} / \Delta\right) \mathrm{S}_{1}\left(\mathrm{Q}_{\mathrm{s}}\right)+$ $+u\left(\lambda_{2} a_{11} / \Delta-\lambda_{1} a_{12} / \Delta\right) S_{2}\left(Q_{s}\right)$

Como $A_{1}=\left(\lambda_{1} a_{22}-\lambda_{2} a_{21}\right)$ e $A_{2}=\left(\lambda_{2} a_{11}-\lambda_{1} a_{12}\right)$ então, uma solução para $\tau_{s}$ é:

$\hat{\tau}_{\mathrm{s}}=\frac{1}{\mathrm{r}(\mathrm{k}-1) \Delta}\left[\frac{\mathrm{k} \Delta}{\mathrm{u}} \mathrm{Q}_{\mathrm{s}}+\mathrm{A}_{1} \mathrm{~S}_{1}\left(\mathrm{Q}_{\mathrm{s}}\right)+\mathrm{A}_{2} \mathrm{~S}_{2}\left(\mathrm{Q}_{\mathrm{s}}\right)\right]$

que é a expressão que permite obter os efeitos ajustados de tratamentos primários para um $\operatorname{PBIB}(2)$. Para maior facilidade de cálculos, quando ocorrer $n_{1}<n_{2}$, tem-se que:

$\hat{\tau}_{s}=\frac{1}{u r(k-1) \Delta}\left[\left(K \Delta-u_{2}\right) Q_{s}+u\left(A_{1}-A_{2}\right) S_{1}\left(Q_{s}\right)\right]$

Uma generalização para a forma matricial $\hat{\tau}=M^{-1} Q$, sendo os elementos da matriz $M^{-1}=$ $\left\{\mathrm{m}_{1 \mathrm{j}}\right\}$ iguais a:

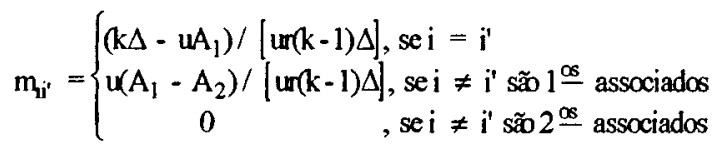

Quando $n_{1}<n_{2}$, tem-se que:

$\hat{\tau}_{s}=\frac{1}{\operatorname{ur}(k-1) \Delta}\left[\left(k \Delta-u A_{1}\right) Q_{s}+u\left(A_{2}-A_{1}\right) S_{2}\left(Q_{s}\right)\right]$

Uma generalização para a forma matricial $\hat{\tau}=M^{-1} Q$, sendo os elementos da matriz $M^{-1}=$ $\left\{m_{1 y}\right\}$ iguais a:

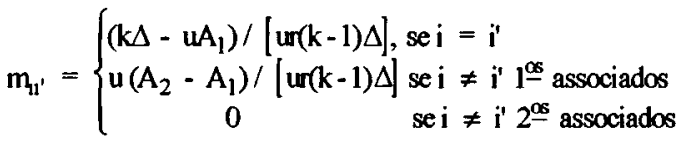

\section{Caso especial: Experimentos em blocos incomple- tos balanceados}

As soluções para os efeitos de tratamentos primários (ajustados) podem ser obtidas, nesse caso, a partir de um delineamento PBIB, considerando-se $\lambda_{1}=\lambda_{2}=\ldots=\lambda_{\mathrm{m}}=\lambda_{\text {Por exemplo, to- }}$ mando-se um PBIB com duas classes de associados, tem-se que:

$a_{11}=\frac{u}{k}[r(k-1)+\lambda]=a_{22} ; \quad a_{12}=a_{21}=0 ;$

$\mathrm{A}_{1}=\mathrm{A}_{2}=\frac{\mathrm{u}}{\mathrm{k}}[\mathrm{r}(\mathrm{k}-1)+\lambda] ; \quad \Delta=\frac{\mathrm{u}^{2}}{\mathrm{k}_{2}}[\mathrm{r}(\mathrm{k}-1)+\lambda]^{2}$

Portanto,

$\hat{\tau}_{\mathrm{s}}=\frac{1}{u r(k-1) \Delta}\left|k \Delta-u A_{2}\right|=\frac{1}{u r(k-1)}\left[k-\frac{u}{\Delta} A_{2}\right] Q_{s}$ mas, lembrando-se que em BIB, tem-se que $\lambda(v-1)=r(k-1)$, segue-se que:

$$
\hat{\tau}_{\mathrm{s}}=\frac{\mathrm{k}}{\lambda \mathrm{vu}} \mathrm{Q}_{\mathrm{s}}
$$

que é a expressão da solução encontrada por IEMMA (1981).

\section{CONCLUSÕES}

1) A solução para os efeitos de tratamentos primários ajustados, foi obtida de modo análogo aquela encontrada para os experimentos em parcelas subdivididas instalados em blocos incompletos balanceados.

2) As soluções para os efeitos referentes aos tratamentos secundários e a interação foram obtidas como nos experimentos com parcelas subdivididas em blocos casualizados.

3) Somente os efeitos de tratamentos primários é que são ajustados para blocos.

\section{REFERÊNCIAS BIBLIOGRÁFICAS}

BOSE, R.C.; NAIR, K.R. Partially balanced incomplete block design. Sankhya, Calcutta, 4: 337-72, 1939. 
CHAKRABARTI, M.C. Mathematics of design and analysis of experiments. Bombay: Asia Publishing House, 1962. 120p.

COCHRAN, W.G.;COX, G.M. Experimental designs. 2 ed. New York: John Wiley, 1957.611p.

DAS, M.N.; GIRI, N.C. Design and analysis of experiments. 2.ed. New York: John Wiley, 1986. 488p.

HICKS, C.R. Fundamental concepts in the design of experiments. 2 ed. New York: Holt, Rinehart an Winston, 1973. 349p.

IEMMA, A.F. Análise de experimentos em parcelas subdivididas com tratamentos principais dispostos em blocos incompletos balanceados. Piracicaba, 1981. 145p. Tese (Doutorado) - Escola Superior de Agricultura "Luiz de Queiroz", Universidade de São Paulo.

IEMMA, A.F. Matrizes para estatística; um texto para profissionais de ciências aplicadas. Piracicaba:ESALQ, Departamento de Matemática e Estatística, 1988. 339p.

JOHN, P.W.M. Incomplete block designs. New York: Marcel Dekker, 1980. $101 \mathrm{p}$.

KEMPTHORNE, $O$. The design and analysis of experiments. New York: John Wiley, 1952.631p.

LEONARD, W.H.; CLARK, A.G. Field plot tecnique. Minneapolis: Burgess, 1939. 288p.
MILLIKEN, G.A ; JOHNSON, D.E. Analysis of messy data; designed experiments. New York: Van Nostrand Reinhold. 1984. 485p.

MORAIS, A.R. de Análise intrablocos de experimentos em parcelas subdivididas com tratamentos principais em blocos incompletos parcialmente balanceados. Piracicaba, 1992. 115p. Tese (Doutorado) - Escola Superior de Agricultura "Luiz de Queiroz"; Universidade de São Paulo.

OLIVEIRA, A.C. de Análise intrablocos de experimentos em blocos incompletos parcialmente balanceados com alguns tratamentos comuns adicionados em cada bloco. Piracicaba, 1985. 153p. Tese (Doutorado)-Escola Superior de Agricultura "Luiz de Queiroz", Universidade de São Paulo.

PIMENTEL GOMES, F. The solution of normal equations of experiments in incomplete blocks. Clêncla e Cultura, São Paulo, 20: 733-46, 1968.

PIMENTEL GOMES, F. Curso de estatística experimental. 11 ed. Piracicaba: Nobel, 1985. 466p.

RAO, C.R. Linear statistical inference and its applications. New York: John Wiley, 1973. 625p.

STEEL, R.G.D. ;TORRIE, J.H. Principles and procedures of statistics. Nova York: McGraw-Hill, 1960. 593p.

Enviado para publicação em 16.03 .93

Aceito para publicação $\mathrm{em} 13.05 .93$ 